\title{
Les problèmes du drainage et de l'irrigation en Lithuanie
}

\author{
M. Rimantes Urbonas \\ Professeur, Recteur de l'Académie Agricole
}

\begin{abstract}
Aloyzes Dirse
Chef de la Chaire de Bonification de l'Académie Agricole de Lithuanie
\end{abstract}

\author{
M. Antanas Maziliauskas
}

Docteur, Président de CNID de Lithuanie

\section{Introduction}

La Lithuanie est un des trois pays Baltes du côté Est de la mer Baltique. La mer influence le climat et par conséquent, le mode de vie. Le total annuel de précipitations est de $625 \mathrm{~mm}$, tandis que l'évaporation est de $390 \mathrm{~mm} / \mathrm{an}$. L'écoulement superficiel ou souterrain s'arrête rapidement après des pluies et les terres deviennent trop humides. Les conséquences négatives peuvent être évitées seulement par le drainage des terres.

On considère qu'en Lithuanie près de $56 \%$ de la surface totale (ou 36,6 mille $\mathrm{km}^{2}$ de la surface totale de 65,2 mille $\mathrm{km}^{2}$ ) sont les terres qui doivent être drainées. Actuellement $80 \%$ de ces terres sont déjà asséchées, dans la majorité des cas par le drainage en tuyaux enterrés.

La distribution des précipitations à travers les mois n'est pas régulière. Les mois de l'été sont très souvent assez secs d'où la nécessité de l'irrigation de certaines cultures, surtout des légumes et des prairies. Actuellement les systèmes d'irrigation sont capables de couvrir une surface d'environ 43 milles ha, ce qui n'est pas suffisant pour les besoins des agriculteurs lithuaniens.

\section{Les problèmes du drainage et de l'irrigation}

Les travaux d'assèchement des terres en Lithuanie ont été commencés bien des années avant la guerre, mais les surfa- ces n'étaient pas importantes et les systèmes d'assèchement n'étaient pas détaillés. Les années 1970-1990 ont été marquées par de vastes travaux de chantier avec équipement de grandes surfaces asséchées. Les entreprises de bonification exécutèrent un total de près de 100000 ha de surface de drainage par an. Avec ces chiffres, au bout des années 1990 on est arrivé à un total de près de 3 millions d'hectares de surfaces drainées.

Mais ces grands travaux n'étaient pas sans risque pour l'environnement. Il y avait des tourbières ou des surfaces aux sols pierreux où l'assèchement s'était fait, le résultat voulu n'a pas été atteint mais les conséquences négatives pour la nature étaient évidentes. La rectification complète de petites rivières et des cours d'eau n'était pas non plus nécessaire. On pouvait certainement recourir à la rectification partielle pour qu'il reste plus de cours d'eau à l'état naturel. Les grandes surfaces des kolkhozes créées pendant les années passées des soviets ainsi que l'utilisation des lourds tracteurs, ont créé certains troubles pour les hydrotechniciens. On était obligé de mettre en jeu des systèmes de drainage de grandes dimensions. Certains parmi eux ont atteint des superficies de 200-300 ha et même plus. Le réseau des canaux à ciel ouvert étant diminué, les conditions pour capter l'eau superficielle sont devenues pires. Les constructions de différents puits ou filtres pour amener l'eau ruisselant sur la surface vers drains et collecteurs n'ont pas donné de résultats satisfaisants.

Ici on peut ajouter le problème de compactage du sol par les lourds tracteurs cultivant la terre. Le non-respect des conditions optimum d'exécution des travaux agricoles et 
les plans gigantesques des kolkhozes d'utilisation des tracteurs de grandes masses pour cultiver la terre, ont donné des conséquences très négatives tant pour la terre ellemême que pour le drainage comme outil de réglage des conditions de vie dans le sol. Dans la majorité des cas, les sols ont été compactés de telle façon que l'eau ne pouvait pas s'infiltrer dans le drainage et celui-ci ne fonctionnait pas, malgré qu'il était en bon état et dans les conditions exigeant son fonctionnement.

Les travaux de reconstruction des systèmes de drainage qui sont menés actuellement, auront pour but non seulement le rétablissement de la capacité des drains eux-mêmes mais aussi le rétablissement de certains réseaux de canaux à ciel ouvert ce qui est possible de nos jours, quand les grandes surfaces kolkhozienes sont divisées en petites surfaces pour des fermiers privés. Il est aussi très important d'ameublir la couche compactée du sol et la stabiliser par voie soit biologique soit chimique.

Nous avons à établir aux plus courts délais les schémas complexes de l'optimisation de l'environnement et de l'aménagement du landscape. Ces chémas pourront être aussi de bons guides pour réaliser les travaux de bonifi- cation dans les années qui viennent. Les travaux des irrigations et du drainage seront en liaison étroite avec la renaturalisation de certains endroits.

Cette année est transitoire pour les agriculteurs lithuaniens. Ils retrouvent la propriété de la terre, mais le problème qui se pose en même temps est, comment le partager pour qu'un système d'irrigation ou de drainage soit la propriété d'une seule personne ? Si avec les systèmes de drainage, il n'y a pas de grands problèmes, l'irrigation n'est pas dans le même cas. Malgré que cet été exigeait d'irriguer au maximum les cultures, surtout les légumes, la moitié des systèmes d'irrigation qui ont été partagés, n'étaient pas en état de fonctionner. Quand on ajoute ici le problème énergétique et la situation actuelle dans la campagne, on peut voir que l'avenir est aux systèmes à la fois plus petits et plus mobiles d'irrigation.

Les recherches dans le domaine du drainage et de l'irrigation sont aussi d'une importance primordiale malgré les difficultés budgétaires des jours actuels, car on ne peut aboutir à une réponse plus ou moins adéquate qu'en ayant des résultats de plusieurs années d'observations. 